\title{
Conical diffraction effect in optical and x-ray Smith-Purcell radiation
}

\begin{abstract}
D. Yu. Sergeeva, ${ }^{*}$ A. A. Tishchenko, ${ }^{\dagger}$ and M. N. Strikhanov
National Research Nuclear University "MEPhI”, Kashirskoe sh. 31, 115409 Moscow, Russia

(Received 4 March 2014; revised manuscript received 12 December 2014; published 8 May 2015)

Smith-Purcell radiation is a well-known phenomenon, which provides a noninvasive scheme for diagnostics of charged particle beams and is used as an effective source of electromagnetic waves, e.g., in the orotron, the free electron laser, etc. In this paper we develop the theory of Smith-Purcell radiation (SPR) for the little-investigated case of arbitrary angles between the charged particle trajectories and the rulings of a grating. The effect of conical diffraction arising here changes drastically the space distribution of the radiation. By contrast to the only existing approach, described by Haeberle et al. [Phys. Rev. E 55, 4675 (1997)], which requires difficult numerical calculations, we give a fully analytic theory of SPR. Also, in this paper we present for the first time the theory of x-ray Smith-Purcell radiation. Evanescent waves on the surface are shown to lead to strong enhancement of Smith-Purcell radiation, through a resonant mechanism. The results are important for the description of real divergent high-brightness beams and for the development of novel noninvasive diagnostic schemes based on the Smith-Purcell effect.
\end{abstract}

DOI: 10.1103/PhysRevSTAB.18.052801

PACS numbers: 42.25.Fx, 41.60.-m, 78.70.-g

\section{INTRODUCTION}

There are two main ways for charged particles to radiate: first, when the particle changes its velocity in an external field. This happens, for example, in Roentgen tubes (bremsstrahlung), in free electron lasers based on undulator radiation, and so on.

The second type of radiation is called polarization radiation and arises when a moving charge acts upon matter by its Coulomb field, and the matter polarized by this dynamically changing field becomes a source of radiation. The charged particle itself can move uniformly and nevertheless generate electromagnetic radiation. Here we talk not only about the widely known Cherenkov and transition radiations, but also about diffraction radiation (DR) (arising when charge moves near a target), about Smith-Purcell radiation (SPR), a special case of DR for targets with periodical surface $[1,2]$, and about parametric $\mathrm{x}$-ray radiation (x-ray radiation of a charged particle moving in a crystal with constant velocity: see, e.g., [3]). Polarization-type interactions of particles with matter also can be a good source of electromagnetic radiation [3-5], including free electron lasers based on SPR [6], but is especially useful for beam diagnostics [2,7].

DR and SPR occupy a special place among the kinds of polarization radiation. They occur when the trajectory of a charged particle is out of the target and the radiation

\section{*DYSergeyeva@mephi.ru \\ Tishchenko@mephi.ru}

Published by the American Physical Society under the terms of the Creative Commons Attribution 3.0 License. Further distribution of this work must maintain attribution to the author $(s)$ and the published article's title, journal citation, and DOI. process is not followed by direct scattering of the particles on the target material. In this case the beam of particles is not disturbed and therefore DR and SPR can provide noninvasive diagnostics of bunches $[2,8]$. SPR was the subject of investigation for many researchers, starting from the original article by Smith and Purcell in 1953 [1]. The theory and applications of SPR are described in monographs $[2,3,9]$. However, the general theory of SPR has not been created yet and at present there exist a number of different approaches, which sometimes are in agreement and sometimes contradict each other. The principal approaches were developed by: (i) van den Berg ([10]: seminumerical, demands difficult numerical calculations); (ii) Shestopalov ([9]: very complicated mathematically, suitable for nonrelativistic beams mainly); (iii) Brownell, Doucas and others ([11]: physically clear and simple, valid mainly for ideally conductive gratings); (iv) Potylitsyn ([2,12], based on results of Kazantsev and Surdutovich for DR [13], only for ideally conductive gratings); (v) Karlovets ([14], the most general theory at present, valid for arbitrary dielectric properties of a target; however, it requires further development and comparison with experimental data).

This short characterization of the methods expresses only our opinion and does not pretend to be an ultimate truth.

For oblique incidence, i.e., at arbitrary angle between particle trajectory and rulings direction, none of the results mentioned above are valid.

Haeberle with coauthors [15] constructed the theory of SPR for the case of oblique incidence as a development of the van den Berg approach, (i) which is very good for nonrelativistic electrons, (ii) which for relativistic electrons sometimes is in good agreement but sometimes disagrees with experiment (see [16] and the discussion in Sec. VA), 
(iii) whose validity in EUV and $\mathrm{x}$-ray range has not been investigated, (iv) and which demands difficult numerical calculations.

In this paper we construct a fully analytical theory of SPR for the case of arbitrary angle between charged particle trajectory and the rulings, from nonrelativistic up to ultrarelativistic particles: (i) in UV and x-ray range, proceeding from the theory of UV and $\mathrm{x}$-ray diffraction radiation created in our previous papers (see [17-19] or in more detail Chapter 4 in [2] and for the bunch of particles in $[20,21])$, (ii) in optics and $\mathrm{THz}$, proceeding from the results for DR of Potylitsyna-Kube and Artru [22], who managed to generalize the theory of Kazantsev and Surdutovich [13].

Why and when can the case of oblique incidence be of interest?

The most important case seems to be that of divergent beams. Coulomb interaction in a beam of moving ultrarelativistic electrons in transversal directions is screened by the magnetic force, and the closer velocity of the electrons to the speed of light, the stronger this suppression is. This effect leads to a weakening of the divergence effects, because the divergence of the beam is caused by the Coulomb repulsion of electrons. Nevertheless, in practice the role of divergence is pronounced in some realistic cases: (a) nonrelativistic beams; (b) moderately relativistic energies for high-brightness beams having small transverse dimensions (compact accelerators for such ranges of energies are now coming into use as effective $\mathrm{x}$-ray and $\mathrm{THz}$ sources in security, industry, medicine etc.); (c) ultrarelativistic high-brightness beams, for example, originating from laser-plasma sources [24]; (d) ultrarelativistic super high-brightness beams for proposed future facilities like ILC, where the size of focal spot is expected to be about $8 \mathrm{~nm}$.

In all these cases conservation of phase volume (Liouville's theorem) results in increasing the divergence, which should be taken into account in a correct theoretical description.

The second situation when oblique incidence can play a vital part arises in (e) the case of a few directions of periodicity, as happens in periodical structures like 2D and

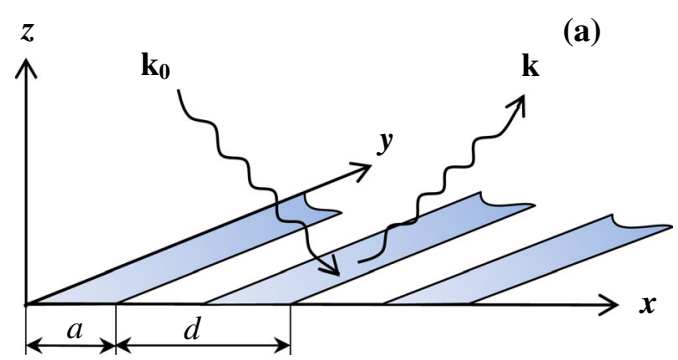

$3 \mathrm{D}$ photonic crystals $[5,23]$. The charged particle moving, e.g., along a 2D or 3D photonic crystal, inside or outside, will produce a radiation distribution of which should be strongly influenced by the conical diffraction.

\section{QUALITATIVE PICTURE OF RADIATION}

In this section we shall demonstrate the analogy between Smith-Purcell radiation and reflection of light from a grating.

The main features of the process of the light reflection from a diffraction grating can be derived from conservation laws.

Photons of light with the wave vector $\mathbf{k}_{0}$ are scattered on some periodical structure (which hereafter we call a diffraction grating, or just a grating) with the period $d$ in the $x$-direction and with rulings along the axis $y$, and becomes the photons of reflected light with the wave vector k, see Fig. 1(a). This process should satisfy the conservation laws:

$$
\begin{aligned}
k_{0 y} & =k_{y}, \\
k_{0 x} & =k_{x}+2 \pi m / d, \quad m=0, \pm 1, \pm 2, \ldots, \\
k_{0} & =k,
\end{aligned}
$$

where $m$ is a diffraction order. The first line expresses the conservation of momentum along the direction of medium homogeneity-axis $y$; the second line states quantified transfer of the momenta along the direction of the periodicity-axis $x$; and the third line implies energy conservation. In the case of $d \rightarrow \infty$ the first and second expressions give us the usual Snell's law. As dielectric properties of the medium where the light is reflected to are not of importance (e.g., one may think that it is air), we put $k_{0}=k=\omega / c$.

Taking into account the relation $\mathbf{k}=(\omega / c) \mathbf{r} / r$ between the vector $\mathbf{k}$ and the radius vector of the point of observation $\mathbf{r}=(x, y, z)$, from the first and third formulas of Eqs. (1) one can obtain the canonical equation for a cone with the main axis $y$ :

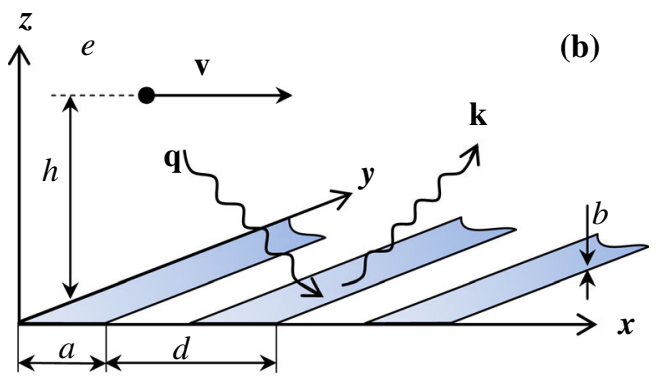

FIG. 1. (a) Reflection of light from a grating. (b) Radiation of a charged particle as a reflection of its own (Coulomb) field from the grating. Velocity of the charged particle is $\mathbf{v}=\left(v_{x}, v_{y}, 0\right), e$ is its charge, the size of a single strip is $(a, \infty, b)$, the grating period is $d, h$ is the impact parameter, i.e., the shortest distance between the grating surface and the particle trajectory; $\mathbf{k}_{0}$ is the wave vector of a photon of incident light, $\mathbf{k}$ is the wave vector of a photon of reflected light, $\mathbf{q}$ is the momentum of virtual photons of the particle Coulomb field. 
(a)
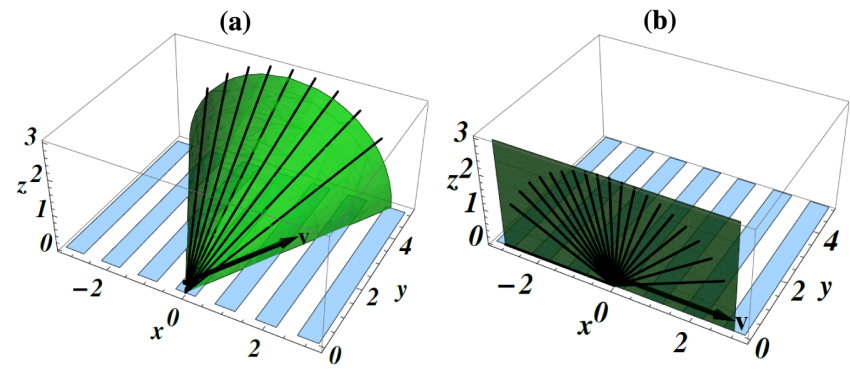

FIG. 2. (a) Conical diffraction at the reflection of monochromatic light from a grating. Separated lines on the conical surface refer to different diffraction orders $m$. (b) Conical diffraction: reducing the cone to a plane in case of $k_{0 y}=0$ (for light reflection) or $\alpha=0$ (for radiation). The angle between velocity $\mathbf{v}$ and axis $x$ is $\alpha$.

$$
\frac{x^{2}}{1-n_{0 y}^{2}}+\frac{z^{2}}{1-n_{0 y}^{2}}-\frac{y^{2}}{n_{0 y}^{2}}=0
$$

where $\mathbf{n}_{0}=\mathbf{k}_{0} / k_{0}$. The reflected light is distributed over the conical surface, so that each diffraction order $m$ is represented by the line on its surface, see Fig. 2(a). In case $k_{0 y}=0$ the cone is reduced to a plane, see Fig. 2(b). The phenomenon of conical diffraction is known in optics [25-27].

Now we consider another process-radiation of a charged particle moving at some distance above the grating, see Fig. 1(b). We expand the Coulomb field $\mathbf{E}_{0}(\mathbf{r}, t)$ into Fourier integral

$$
\mathbf{E}_{0}(\mathbf{r}, t)=\int \frac{d^{3} r}{(2 \pi)^{3}} \int \frac{d t}{2 \pi} e^{-i \mathbf{q} \cdot \mathbf{r}} e^{i \omega t} \mathbf{E}_{0}(\mathbf{q}, \omega)
$$

For a charge $e$ moving with the constant velocity $\mathbf{v}=$ $\left(v_{x}, v_{y}, 0\right)$ at the distance $h$ (impact parameter) above the plane $z=0$ we have

$$
\mathbf{E}_{0}(\mathbf{q}, \omega)=-\frac{i e}{2 \pi^{2}} \frac{\mathbf{q}-\mathbf{v} \omega / c^{2}}{q^{2}-k^{2}} e^{-h \rho} \delta(\omega-\mathbf{q} \cdot \mathbf{v})
$$

Considering reflection of a plane wave (Fourier component) $\exp (-i \mathbf{q} \cdot \mathbf{r})$ from the grating with the period $d$ in the $x$-direction one can write the conservation laws as in Eq. (1):

$$
\begin{aligned}
q_{y} & =k_{y}, \\
q_{x} & =k_{x}+2 \pi m / d, \quad m=1,2, \ldots \\
q & =k
\end{aligned}
$$

The vector $\mathbf{q}$ in Eq. (5) is unknown, and besides these equations do not contain the characteristics of the charged particle. So, one should redefine this system using one more equation, namely,

$$
\omega=\mathbf{q} \cdot \mathbf{v}
$$

which comes from the delta function in Eq. (4). Noting that $q_{z}=\sqrt{q^{2}-q_{x}^{2}-q_{y}^{2}}$, from Eqs. (5) and (6) one can get

$q_{z}=i \rho, \quad \rho=\sqrt{\left(\frac{\omega-k_{y} v_{y}}{v_{x}}\right)^{2}+\left(k_{y}\right)^{2}-\left(\frac{\omega}{c}\right)^{2}}$.

Exponential decreasing of the Coulomb field of the particle with rise of impact-parameter $h$ results in the same decreasing of the radiation filed generated by the field from Eq. (4), which is a characteristic feature of Smith-Purcell radiation. The radiation is maximal when $\rho$ is minimal:

$$
\rho_{\min }=\frac{\omega}{c \beta \gamma}
$$

which occurs at

$$
k_{y}=\frac{\omega}{c} \frac{\beta_{y}}{\beta^{2}}
$$

where $\boldsymbol{\beta}=\mathbf{v} / c$. Using the first and second expressions from Eq. (5) we can rewrite Eq. (6) in the form

$$
\omega=v_{x}\left(k_{x}+2 \pi m / d\right)+v_{y} k_{y} .
$$

This is Smith-Purcell dispersion relation in its general form; it interlinks the direction of observation $\mathbf{n}=\mathbf{k} / k$, wavelength $\lambda=2 \pi c / \omega$, velocity $\mathbf{v}$ and the period of a grating $d$.

For $v_{y}=0 \mathrm{Eq}$. (10) gives the well-known relation obtained by Smith and Purcell [1]:

$$
\frac{1}{\beta}-n_{x}=\frac{\lambda}{d} m, \quad m=1,2, \ldots
$$

One can see from Eq. (9) that $k_{y}=0$ if $v_{y}=0$, and therefore a maximum of radiation is in the plane $y=0$, see Fig. 2(b).

With the help of Eq. (9), as it was in the case with light reflection, we obtain the equation of a cone:

$$
\frac{x^{2}}{1-\beta_{y}^{2} / \beta^{4}}+\frac{z^{2}}{1-\beta_{y}^{2} / \beta^{4}}-\frac{y^{2}}{\beta_{y}^{2} / \beta^{4}}=0 .
$$

This equation resembles Eq. (2) with the only difference: characteristics of "incident light" are defined by properties of the charged particle, so that $\beta_{y} / \beta^{2}$ plays the role of $n_{0 y}$.

So, the spatial distribution of Smith-Purcell radiation at oblique incidence $\left(\beta_{y} \neq 0\right)$ is close to that for the conical diffraction of light: in accordance with Eqs. (10) and (12) the radiation is distributed over the conical surface [given by Eq. (12)] as separate peaks with different orders $m=1,2, \ldots$. This qualitative analysis shows the principal 
features of radiation, but is insufficient to catch all differences between SPR and conical diffraction of light. Below we shall draw analytical expressions and then analyze the conical diffraction effect in Smith-Purcell radiation in more detail.

\section{X-RAY SMITH-PURCELL RADIATION}

In this section we construct the theory of Smith-Purcell radiation at frequencies

$$
\omega \gg \omega_{p} .
$$

Here $\omega_{p}$ is the plasma frequency, which usually has values about $20-30 \mathrm{eV}$ [28].

In practice, inequality

$$
\omega \geq 3 \omega_{p}
$$

is enough. Therefore, this approach works for the wavelengths

$$
\lambda \leq 2 \pi \frac{66 \mathrm{~nm}}{\omega_{p}(\mathrm{eV})}
$$

where $\omega_{p}$ is taken in electron-volts and $\lambda$ is measured in nanometers. For example, for $\omega_{p}=24,4 \mathrm{eV}$ (Mylar, [28]) we have $\lambda \leq 17 \mathrm{~nm}$, for $\omega_{p}=13,8 \mathrm{eV}$ (lithium, [28]) $\lambda \leq 30 \mathrm{~nm}$. Hereafter talking of EUV/x-ray range we shall mean frequencies satisfying Eq. (13) or (14).

We discussed in our previous article [17] that, constructing the correct theory of DR and SPR, it is appropriate to use the method applied by Durand [29] for calculation of x-ray transition radiation and based (i) on consideration of propagation of the radiation inside a medium and (ii) on separate analysis of the process of refraction at the surface. Indeed, the refraction was neglected in [29]. As we proved later, this is correct only when we deal with one charged particle and simple interface; for the beams or for periodic structures the refraction of radiation at the surface of the target should be taken into account. The theory of x-ray DR was developed by this method in the papers [2,17-19,30] for the single-particle case, and in papers [20,31] for bunches of charged particles; for optical range this approach was developed in papers [14,32,33].

For x-ray region the dielectric function of a medium $\varepsilon(\omega)$ can be written as

$$
\begin{aligned}
\varepsilon(\omega) & =1+\chi^{\prime}(\omega)+i \chi^{\prime \prime}(\omega), \\
\chi^{\prime}(\omega) & =-\omega_{p}^{2} / \omega^{2} .
\end{aligned}
$$

For the sake of simplicity we shall neglect absorption, i.e., take $\chi^{\prime \prime} \ll\left|\chi^{\prime}(\omega)\right|$ below; the expansion of the theory is possible as in paper [19], where $\mathrm{x}$-ray Cherenkov radiation in conditions of diffraction radiation (i.e., when the trajectory of a particle does not cross the target) was investigated. Note that in region $\omega \gg \omega_{p}$ the value of $\varepsilon(\omega)$ is close to the unity, and therefore any models dealing with ideally conducting targets [15,34] are not applicable for describing of UV and x-ray Smith-Purcell and diffraction radiation.

Let the charge $e$ move with the constant velocity $\mathbf{v}=$ $\left(v_{x}, v_{y}, 0\right)$ at a distance $h$ from the target surface (impact parameter)—see Fig. 1(b). The target is a grating consisting of $N$ strips with period $d$ and vacuum between strips (air in practice). The size of a strip is $(a, \infty, b)$.

The Coulomb field of the charged particle decreases with increase in impact-parameter $h$ in the direction to the target surface and the attenuation distance is $\gamma \beta \lambda / 2 \pi$ - see Eqs. (4) and (5), $\gamma=E / M c^{2}$ is the Lorenz factor which equals the particle energy $E$ divided by the energy of a particle at rest $M c^{2}$.

Thus, it is easy to estimate the minimal energy of electrons for which the radiation is intensive enough to be observed in the frequency range $\omega \gg \omega_{p}$ :

$$
E>E_{\min }=2 \pi h / \beta \lambda(\mathrm{MeV}) .
$$

Estimating the minimal frequency as $\omega=3 \omega_{p}=78.3 \mathrm{eV}$ (beryllium [28]), which corresponds to the wavelength $\lambda_{\max }=15.7 \mathrm{~nm}$, we obtain

$$
E>(h \mu \mathrm{m})\left(4 \times 10^{2} \mathrm{MeV}\right),
$$

where $h$ is measured in micrometers, $E$ in megaelectronvolts. The value of impact-parameter $h$ is bounded from below by the beam size. Usually the transversal size of ultrarelativistic beams is of the order of $10 \mu \mathrm{m}$ and less. Estimating the minimal value of $h$ being about $10 \mu \mathrm{m}$, one can see that the energy of electrons $E$ should exceed $4 \mathrm{GeV}$ in order to DR and SPR in EUV and x-ray range would be appreciable.

The Coulomb field of a moving charged particle produces dynamic polarization currents in medium. This leads to arising of the radiation determined by Fourier image of the current density $\mathbf{j}(\mathbf{r}, \omega)$ :

$$
\mathbf{j}(\mathbf{r}, \omega)=\frac{\omega}{4 \pi i}[\varepsilon(\omega)-1] \mathbf{E}_{0}(\mathbf{r}, \omega) .
$$

The Coulomb field of moving charge in vacuum $\mathbf{E}_{0}(\mathbf{r}, \omega)$ can be found from Eqs. (3) and (4). Note, we take the expression for the Coulomb field in vacuum-to take it in form including contribution of polarization of medium would be incorrect, see the discussion in Chapter 4 of monograph [2].

The radiation field is determined by current density as

$$
\mathbf{E}^{\prime}(\mathbf{r}, \omega)=\frac{i \omega}{c^{2}} \frac{e^{i k^{\prime} r}}{r}\left(\mathbf{n}^{\prime} \times \mathbf{n}^{\prime} \times \int_{V} d^{3} r \mathbf{j}(\mathbf{r}, \omega) e^{-i \mathbf{k}^{\prime} \cdot \mathbf{r}}\right),
$$


where it is integrated over the region of existence of polarization currents, i.e., over the target volume $V$; $\mathbf{k}^{\prime}=\mathbf{n}^{\prime} \sqrt{\varepsilon(\omega)} \omega / c$ is the wave vector inside the medium [2]. The prime means that a variable is taken inside the matter.

Equations (19), (20) and (4) permit one to calculate the radiation field inside the medium:

$$
\begin{aligned}
\mathbf{E}^{\prime}(\mathbf{r}, \omega)= & -\frac{e^{i k^{\prime} r}}{r} \frac{e}{4 \pi} \frac{\varepsilon(\omega)-1}{v_{x}} e^{-\rho^{\prime} h} \frac{\omega^{2}}{c^{2}}\left(1-e^{-b \rho^{\prime}+i k_{z}^{\prime} b}\right) \\
& \times\left[\mathbf{n}^{\prime} \times \mathbf{n}^{\prime} \times\left(\frac{\mathbf{A}^{\prime}}{\rho^{\prime}}-i \mathbf{e}_{z}\right)\right] \\
& \times \frac{1}{\rho^{\prime}-i k_{z}^{\prime}} \sum_{s=1}^{N}\left(e^{i \varphi^{\prime} s d} \frac{e^{i \varphi^{\prime} a}-1}{i \varphi^{\prime}}\right) .
\end{aligned}
$$

Here

$$
\begin{aligned}
\mathbf{A}^{\prime} & =\frac{\omega-k_{y}^{\prime} v_{y}}{v_{x}} \mathbf{e}_{x}+k_{y}^{\prime} \mathbf{e}_{y}-\mathbf{v} \frac{\omega}{c^{2}}, \\
\varphi^{\prime} & =\left(\omega-k_{x}^{\prime} v_{x}-k_{y}^{\prime} v_{y}\right) / v_{x}, \\
\rho^{\prime 2} & =\left(\frac{\omega-k_{y}^{\prime} v_{y}}{v_{x}}\right)^{2}+\left(k_{y}^{\prime}\right)^{2}-\left(\frac{\omega}{c}\right)^{2} .
\end{aligned}
$$

Generally speaking, the radiation can be refracted and reflected at the upper and forward facets (sides). In the frequency region $\omega \gg \omega_{p}$ one can neglect the reflection dealing with a single particle [17-19,29]. However, when interference and coherence phenomena can take place, the weak effects can be accumulated, and taking the refraction into account is important [20]. As we consider the radiation from a grating, i.e., periodical structure, the interference occurs, and refraction at the facets ought to be taken into account. We consider the situation when polarization currents are produced in a thin layer near the upper facet of the target. When

$$
\begin{aligned}
& n_{z}^{\prime} \gg n_{x}^{\prime} \frac{b^{*}}{a}, \\
& b^{*}=\min \left\{\frac{\gamma \beta \lambda}{2 \pi}-h, b\right\},
\end{aligned}
$$

where $b^{*}$ is the thickness of effectively excited and radiating part of the target, then the radiation goes out in vacuum only through the upper facet.

If Eq. (23) is not satisfied, then results will be correct only qualitatively; to obtain more accurate results one needs to take into account the refraction of the radiation at the forward facet. The case when radiation goes out through both upper and forward facets was analyzed in [30].

Applying the law of refraction for the upper facet we get the connection between the unit wave vector in the matter $\mathbf{n}^{\prime}$ and the unit wave vector in the vacuum $\mathbf{n}$ in the form

$$
\sqrt{\varepsilon(\omega)} \mathbf{n}^{\prime}=\left(n_{x}, n_{y}, \sqrt{\varepsilon(\omega)-1+n_{z}^{2}}\right) .
$$

We would like to stress that at

$$
n_{z}^{\min }=\sqrt{1-\varepsilon(\omega)}
$$

the $z$-component of the wave vector in medium $k_{z}^{\prime}$ becomes imaginary, which corresponds to the evanescent wave $\exp \left(z k_{z}^{\prime}\right), z<0$. These waves turn to be the radiation field $\exp \left(-i z k_{z}\right), z>0$, out of the medium, see Fig. 3. Usually, talking of the evanescent surface waves one means the waves decreasing exponentially on both sides of the boundary. Contrary to that, the radiation under the angles less than the angles satisfying Eq. (25) proves to be described by rather unusual waves which decrease exponentially on the one side of the boundary $\left(\exp \left(-|z| k_{z}^{\prime}\right)\right.$, $z<0)$ and are outgoing plane waves $\left(\exp \left(-i z k_{z}\right), z>0\right)$ on the other side of the boundary, see Fig. 3(b). This solution of Maxwell equations, as far as we know, for the first time was described by Brekhovskikh [35].
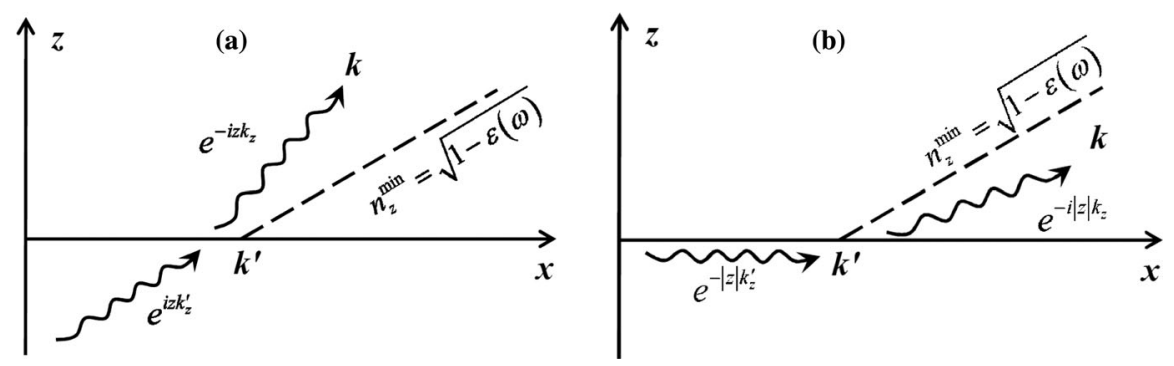

FIG. 3. X-ray radiation leaves the target refracting at its upper facet: (a) the "volume" wave exp $\left(i z k_{z}^{\prime}\right), z<0$, refracts at the surface and goes out at the angle more than $\theta_{c r}$ that is defined by Eq. (25); (b) the evanescent wave $\exp \left(-|z| k_{z}^{\prime}\right), z<0$, gives the radiation $\left(\exp \left(-i z k_{z}\right), z>0\right)$ at the angle less than $\theta_{c r}$. 
So, the radiation field outside the medium has the form

$$
\begin{aligned}
\mathbf{E}(\mathbf{r}, \omega)= & -\frac{e}{4 \pi} \frac{\varepsilon(\omega)-1}{v_{x}} e^{-\rho h} \frac{\omega^{2}}{c^{2}} \frac{e^{i k r}}{r} \frac{\mathbf{n}^{\prime} \times \mathbf{n}^{\prime} \times\left(\mathbf{A} / \rho-i \mathbf{e}_{z}\right)}{\rho-i \frac{\omega}{c} \sqrt{\varepsilon(\omega)-1+n_{z}^{2}}} \\
& \times\left[1-\exp \left(-b \rho+i b \frac{\omega}{c} \sqrt{\varepsilon(\omega)-1+n_{z}^{2}}\right)\right] \\
& \times \frac{e^{i \rho a}-1}{i \varphi} \sum_{s=1}^{N} e^{i \varphi s d}
\end{aligned}
$$

Here

$$
\begin{aligned}
\varphi & =\frac{\omega}{c} \frac{1}{\beta_{x}}\left(1-n_{x} \beta_{x}-n_{y} \beta_{y}\right), \\
\rho & =\frac{\omega}{c \beta \gamma} \sqrt{1+\gamma^{2} \beta_{x}^{-2}\left(n_{y} \beta^{2}-\beta_{y}\right)^{2}}, \\
\mathbf{A} & =\frac{\omega}{\beta_{x} c}\left(1-n_{y} \beta_{y}-\beta_{x}^{2}, n_{y} \beta_{x}-\beta_{x} \beta_{y}, 0\right),
\end{aligned}
$$

and $\mathbf{n}^{\prime}$ is taken from the right part of Eq. (24).

Using Eq. (26) it is easy to find the energy of SmithPurcell radiation emitted per unit solid angle and frequency in EUV/X-ray range:

$\frac{d^{2} E(\mathbf{n}, \omega)}{d \Omega d \hbar \omega}=\frac{d^{2} E_{0}(\mathbf{n}, \omega)}{d \Omega d \hbar \omega} 4 \sin ^{2}\left(\frac{a \varphi}{2}\right) \frac{\sin ^{2}(N d \varphi / 2)}{\sin ^{2}(d \varphi / 2)}$.

Here expression

$$
\begin{aligned}
\frac{d^{2} E_{0}(\mathbf{n}, \omega)}{d \Omega d \hbar \omega}= & \frac{1}{137}\left(\frac{\varepsilon(\omega)-1}{4 \pi \beta_{x} \varphi}\right)^{2} e^{-2 \rho h} F_{b} \frac{\omega^{4}}{c^{4}} \\
& \times \frac{\left|\left[\mathbf{n}^{\prime} \times \mathbf{n}^{\prime} \times\left(\mathbf{A} / \rho-i \mathbf{e}_{z}\right)\right]\right|^{2}}{\left|\rho-i(\omega / c) \sqrt{\varepsilon(\omega)-1+n_{z}^{2}}\right|^{2}}
\end{aligned}
$$

describes the radiation from one strip (slab) with sizes $(a, \infty, b)$, factor $F_{b}$ describes the dependence of radiation on the strip thickness:

$F_{b}=\left|1-\exp \left(-b \rho+i b \frac{\omega}{c} \sqrt{\varepsilon(\omega)-1+n_{z}^{2}}\right)\right|^{2}$.

Equation (28) in the particular case of $N=1$ coincides with the results of paper [17], when corrections of the order of $\left(\omega_{p} / \omega\right)^{2} \ll 1$ and $\gamma^{-2} \ll 1$ are neglected. In the case of $\beta_{y}=0(\alpha=0)$ the results are similar to those reported in [36]. Close results for X-ray SPR at $\alpha=0$ and small angles of radiation were obtained in [37].

\section{SMITH-PURCELL RADIATION AT OPTICAL AND LOWER FREQUENCIES}

Now we shall consider the radiation from $N$ thin strips (thickness $b \rightarrow 0$ ) for those very configuration as in Sec. III [see Figs. 1(b) and 2(a)], but at the optical and lower frequencies. We shall proceed from the results of Potylitsyna-Kube and Artru [22], who managed to generalize the well-known theory of diffraction radiation for infinitely thin and ideally conducting half-plane-the model created by Kazantsev and Surdutovich [13] using the Wiener-Hopf technique.

The expression for the field of the radiation $\mathbf{E}_{H}(\mathbf{r}, \omega)$ from an ideally conducting half-plane can be obtained from the results of [22]. Let us consider the target as a grating of $N$ strips of width $a$, see Fig. 4(a). Each strip has two edges, therefore the resulting radiation can be obtained from simple geometrical consideration. The phase difference $\Delta_{a}=\mathbf{k} \Delta \mathbf{r}-\omega \Delta t$ of the waves generated by each edge of the strip is written in the form

$$
\Delta_{a}=\frac{\omega}{c} \frac{a}{\beta_{x}}(\boldsymbol{\beta} \mathbf{n}-1) .
$$

The field of radiation from a single strip in case $a \gg \lambda$ is

$$
\mathbf{E}_{a}(\mathbf{r}, \omega)=\mathbf{E}_{H}(\mathbf{r}, \omega)-\mathbf{E}_{H}(\mathbf{r}, \omega) \exp \left(i \Delta_{a}\right) .
$$

Terms in Eq. (32) have opposite signs because for the first edge the particle flies coming to the strip, and for the second edge it flies getting away.

Writing the analogous expressions for each of $N$ strips

$$
\begin{gathered}
\Delta_{N}=\frac{\omega}{c} \frac{d}{\beta_{x}}(\boldsymbol{\beta n}-1), \\
\mathbf{E}_{N}(\mathbf{r}, \omega)=\mathbf{E}_{a}(\mathbf{r}, \omega)+\mathbf{E}_{a}(\mathbf{r}, \omega) \exp \left(i \Delta_{N}\right)+\cdots \\
+\mathbf{E}_{a}(\mathbf{r}, \omega) \exp \left[i(N-1) \Delta_{N}\right],
\end{gathered}
$$

(a)

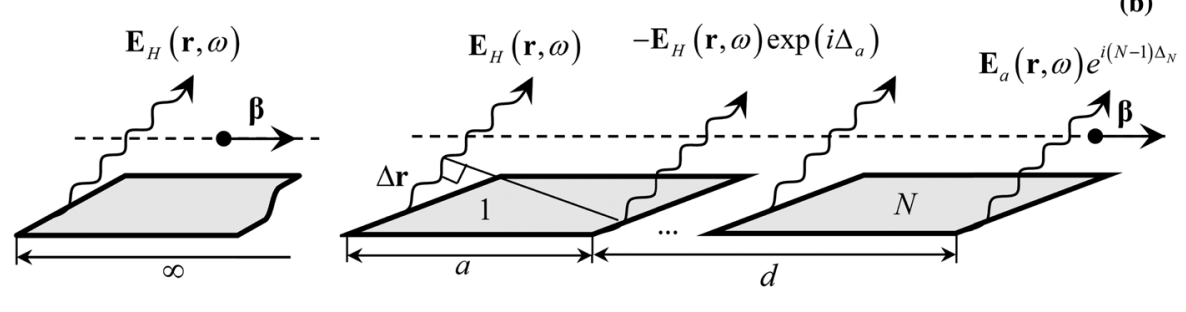

FIG. 4. Scheme of generating of optical SPR. 
we can find the expression for distribution of emitted energy of SPR in the form of Eq. (28), where $d^{2} E_{0}(\mathbf{n}, \omega) /(d \Omega d \hbar \omega)$ is found from [22] after adapting it to the coordinate system taken in our paper:

$$
\begin{aligned}
\frac{d^{2} E(\mathbf{n}, \omega)}{d \hbar \omega d \Omega}= & \frac{1}{137} \frac{1}{4 \pi^{2}} \frac{\beta_{x}\left(1-\beta_{y} n_{y}\right)}{\sqrt{1-n_{y}^{2}}}\left(\frac{\omega}{c \beta_{x}}\right)^{4} \frac{1}{\rho^{2} \varphi^{2}} \exp (-2 \rho h) \\
& \times\left[\left(1-2 \beta_{y} n_{y}-\beta_{x}^{2}+\beta^{2} n_{y}^{2}\right)\left(1+\frac{\beta_{x} \sqrt{1-n_{y}^{2}}}{1-\beta_{y} n_{y}}\right)\left(1-\frac{n_{x}}{\sqrt{1-n_{y}^{2}}}\right)\right. \\
& \left.+\left(\beta_{y}-n_{y}\right)^{2}\left(1-\frac{\beta_{x} \sqrt{1-n_{y}^{2}}}{1-\beta_{y} n_{y}}\right)\left(1+\frac{n_{x}}{\sqrt{1-n_{y}^{2}}}\right)\right] 4 \sin ^{2}\left(\frac{a \varphi}{2}\right) \frac{\sin ^{2}(N d \varphi / 2)}{\sin ^{2}(d \varphi / 2)}
\end{aligned}
$$

Here $\rho=\frac{\omega}{c \beta \gamma} \sqrt{1+\gamma^{2} \beta_{x}^{-2}\left(n_{y} \beta^{2}-\beta_{y}\right)^{2}}, \varphi=\frac{\omega}{c} \frac{1}{\beta_{x}}\left(1-n_{x} \beta_{x}-\right.$ $n_{y} \beta_{y}$ ) are the same as in the $\mathrm{x}$-ray range. Equation (35) in the case of $\alpha=0$ coincides with the expression obtained earlier [2], and for $N=1$ coincides with the results of papers $[17,12]$.

\section{ANALYSIS}

\section{A. Spatial distribution of Smith-Purcell radiation}

According to Eqs. (28) and (35) the distribution of the radiation both in $\mathrm{x}$-ray and optical regions is proportional to the exponent and the ratio of squared sines:

$$
\frac{d^{2} E(\mathbf{n}, \omega)}{d \Omega d \hbar \omega} \propto \frac{\sin ^{2}(N d \varphi / 2)}{\sin ^{2}(d \varphi / 2)} \exp (-2 \rho h) .
$$

These are two multipliers that define the surface of maximal SPR. When the target consists of many strips, i.e., $N \gg 1$, the ratio of squared sines goes to the sum of delta functions:

$$
\frac{\sin ^{2}(N d \varphi / 2)}{\sin ^{2}(d \varphi / 2)} \stackrel{N \gg 1}{\rightarrow} 2 \pi N \sum_{m} \delta(d \varphi-2 \pi m) .
$$

The Smith-Purcell dispersion relation in the case of oblique incidence follows from Eqs. (37) and (27):

$$
\frac{d}{\lambda} \frac{1}{\beta_{x}}\left(1-n_{x} \beta_{x}-n_{y} \beta_{y}\right)=m, \quad m=1,2, \ldots
$$

Designating the angle between the velocity and the direction of radiation as $\chi$ we write Eq. (38) in the form

$$
\frac{d}{\cos \alpha}\left(\beta^{-1}-\cos \chi\right)=\lambda m, \quad m=1,2, \ldots
$$

Index $m$ is the diffraction order. Equation (38) coincides with Eq. (10) obtained with the help of conservation laws.
Equation (39) was obtained also in works [15,38]. In [38], the authors got it, but did not analyze. In [15] the effect of conical diffraction in SPR was considered for low frequency range and for nonrelativistic particles only. We would like to notice that the model of van den Berg [10] followed by the authors of paper [15] can be applied also for the relativistic case, but with variable success. For example, see the comparison with experimental data for energies of electrons of $855 \mathrm{MeV}$ in the paper of Kube et al. [16], where, comparing theory and experiment, in Fig. 11 one can see rather good agreement (deep grating), in Fig. 10 for deep grating the agreement is satisfactory for $\lambda=360 \mathrm{~nm}$ and bad for $\lambda=546 \mathrm{~nm}$ (discrepancy in ten times), and in Fig. 12 contradiction between theory and experiment for shallow grating (discrepancy in hundred times). In paper [16] another problem of the van den Berg theory was also mentioned: it requires extensive numerical calculations until finally the numerical solution of the integral equations converges, which is especially hard in the case $\lambda \ll d$ (see also [39]). The interested reader can find in paper [40] the comparison the theories of van den Berg [10], Brownell, Doucas and others [11], and Potylitsyn [2,12]; see also the short discussion in the Introduction.

The second multiplier $\exp (-2 \rho h)$ in Eq. (36) has maximum when $\rho=\rho_{\min }$, which gives the condition

$$
n_{y}^{\max }=\beta_{y} / \beta^{2} .
$$

It follows from Eqs. (38) and (40) that the Smith-Purcell peaks, corresponding to different values of the spectral order $m$, in the case of oblique incidence are distributed over the surface of the cone, centered along the axis $y$ (i.e., the axis of rulings direction), like it was shown in Sec. II. Choosing $\beta_{x}=\beta \cos \alpha, \beta_{y}=\beta \sin \alpha$ we find the equation of the cone, agreeing with Eq. (12):

$$
\frac{x^{2}}{1-\frac{\sin ^{2} \alpha}{\beta^{2}}}+\frac{z^{2}}{1-\frac{\sin ^{2} \alpha}{\beta^{2}}}-\frac{y^{2}}{\frac{\sin ^{2} \alpha}{\beta^{2}}}=0 .
$$




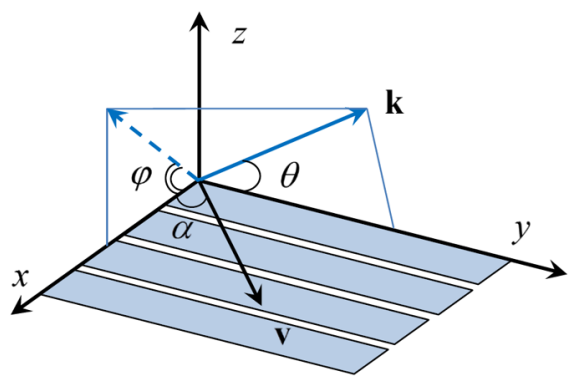

FIG. 5. Schematic convention of the axes.

From Eqs. (38) and (40) we can find the angles describing positions of peaks on the cone. Assuming $\theta$ is polar angle and $\phi$ is the azimuthal angle as it is shown in Fig. 5,

$$
\begin{aligned}
& n_{x}=\sin \theta \cos \phi, \\
& n_{y}=\cos \theta, \\
& n_{z}=\sin \theta \sin \phi,
\end{aligned}
$$

one can obtain a set of couples $\left\{\theta_{m}, \phi_{m}\right\}$ - the angles defining the direction of maximal radiation corresponding to the $m$ th diffraction order:

$$
\left\{\begin{array}{l}
\theta_{m}=\arccos \left(\beta^{-1} \sin \alpha\right) \\
\phi_{m}=\arccos \left(\frac{1}{\beta} \frac{\cos \alpha-\lambda m \beta / d}{\sqrt{1-\left(\beta^{-1} \sin \alpha\right)^{2}}}\right), \quad m=1,2, \ldots
\end{array}\right.
$$

It follows from Eqs. (43) and (42) that for orders

$$
m \geq \frac{d}{\lambda} \frac{\cos \alpha}{\beta}
$$

the radiation is distributed in half-space with negative value of $x$. Diffraction and transition radiations are usually thought to be concentrated near the direction of the charged particle velocity. The radiation propagating in the direction of mirror reflection of particle velocity relative to the rulings direction is called "backward radiation"-_backward TR or backward DR. As opposed to them, SPR represents the peaks (rays) of radiation distributed in space over the conical surface. Let us find a condition of existing of a SPR peak in the direction of mirror reflection relative to the rulings direction for each SPR peak. First, we write the dispersion relation Eq. (38) for the peak of SPR of $m_{1}$ order in the "forward" direction, i.e., for $n_{x}>0$; and for the peak of SPR of $m_{2}$ order in the direction of mirror reflection to $m_{1}$, i.e., for $n_{x}<0$. Summing up these two equations we find

$$
2 \frac{d}{\lambda} \frac{1}{\beta_{x}}\left(1-n_{y} \beta_{y}\right)=m_{1}+m_{2} .
$$

Designating $m_{2}+m_{1}=l, l=2,3, \ldots$, with help of the condition for the cone $n_{y}=\beta_{y} / \beta^{2}$ one can see that in the case of

$$
\frac{d}{\lambda}=\frac{\beta}{2 \cos \alpha} l, \quad l=2,3 \ldots
$$

there is a peak in the direction of mirror reflection $(x \rightarrow-x)$ for each peak (spectral diffraction order) on the conical surface.

Except for multipliers analyzed in this section, see Eq. (36), the distributions given by Eqs. (28) and (35) contain the additional multiplier which depends on the angles of radiation. This leads to decreasing of the intensity of the peaks and their shifting, which we consider below.

\section{B. Spectral and angular distribution of Smith-Purcell radiation}

Let us consider the distributions of the radiation obtained in Secs. III and IV, see Eqs. (28) and (35). Figures 6-8 demonstrate EUV/x-ray, optical and $\mathrm{THz}$ radiation distributions on the conical surface, i.e., for the fixed angle $\theta=\arccos \left(\beta^{-1} \sin \alpha\right)$.

Figure 6 shows the distribution of x-ray SPR for two values of $\alpha$. Equation (23) gives the value of the angle dividing the angular region into two parts-I and II in Fig. 6. The value of this angle is shown as thick vertical tall lines (black and dashed red). For the angles in part I [i.e., the region where Eq. (23) is not satisfied] our results should be considered as only qualitative, because these do not take into account the refraction of radiation going out through the front facet of the target. For the angles in part II [or region where Eq. (23) is satisfied] the results are exact.

It follows from Eq. (16) that in the EUV/x-ray frequency region any substance is optically less dense than vacuum.

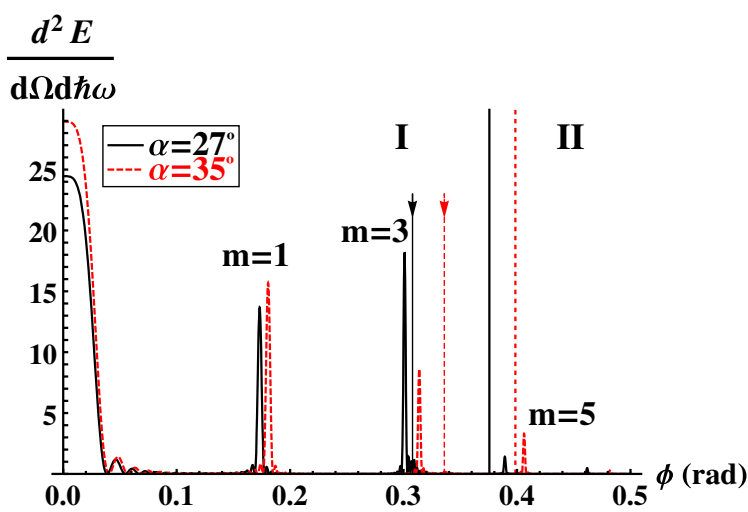

FIG. 6. The distribution of x-ray SPR over the conical surface, i.e., at $\theta=\arccos \left(\beta^{-1} \sin \alpha\right)$ [see Eq. (28)]. Here $\gamma=4 \times 10^{4}$ (electron energy of FACET, SLAC: $E=28 \mathrm{GeV}$ ), $\hbar \omega_{p}=$ $26.1 \mathrm{eV}$ (beryllium), $d=0.9 \mu \mathrm{m}, a=0.45 \mu \mathrm{m}, b=0.1 \mu \mathrm{m}$, $\lambda=12 \mathrm{~nm}, h=60 \mu \mathrm{m}, \varepsilon(\omega)=1-\omega_{p}^{2} / \omega^{2}, N=20$. For the black curve $\alpha=27^{\circ}$, for the dashed red curve $\alpha=35^{\circ}$. The thick tall lines correspond to the value dividing angles into two parts-I and II, where Eq. (23) is satisfied and not satisfied, respectively. The thin lines with arrows correspond to the $\phi_{\min }$ from Eq. (25) or Eq. (47). For the black curve $\phi_{\min } \approx 0.31 \mathrm{rad}$, for the red dashed curve $\phi_{\min } \approx 0.34 \mathrm{rad}$. 
Therefore, the minimal angle of refraction exists that can be obtained from Eqs. (25) and (42) for real $n_{z}^{\prime}$ :

$$
\phi>\phi_{\min }, \quad \sin \phi_{\min }=\frac{\sqrt{1-\varepsilon(\omega)}}{\sin \theta} .
$$

In the general case, when we are not restricted by Eq. (47), there is a narrow high peak described by the multiplier in Eq. (28):

$$
\left|\rho-i \frac{\omega}{c} \sqrt{\varepsilon(\omega)-1+n_{z}^{2}}\right|^{-2} .
$$

Actually, Eq. (48) has maximum when $\varepsilon(\omega)-1+n_{z}^{2}=0$ and when Eq. (40) is fulfilled. For the sake of clarity, we show the position of this peak in Fig. 6 as the thin vertical lines with arrows [see also peaks in Fig. 9(b)]. This peak corresponds to forward diffraction radiation rather than SPR. By its nature it is defined by the (evanescent) waves which we discussed after Eq. (25). Using Eq. (48) it is not hard to estimate that the half-width of this peak (at $\gamma \gg 1$, $\left.\omega \gg \omega_{p}\right)$ is

$$
\delta \tilde{\phi}=0.6 \frac{\omega}{\omega_{p}} \frac{\gamma^{-2}}{\cos \alpha}
$$

where it is supposed that the reasonable conditions $\omega_{p}<\omega<\gamma \omega_{p}, \pi / 2-\alpha \gg \gamma^{-1}$ are fulfilled.

It is important that this peak can influence on the intensity of SPR. In Fig. 6 for $\alpha=27^{\circ}$ (black curve) this peak and the SPR peak of the third order $(m=3)$ are close to each other and as a result the intensity of the third SPR peak is considerably higher than the expected one. For $\alpha=35^{\circ}$ (red dashed curve) this peak is situated far from the SPR peaks and its influence is insignificant.

Both for optical and for $\mathrm{THz}$ regions the intensities of radiation slowly decrease from the first to last diffraction orders. Notice that intensity of radiation at $\phi=0$ equals zero (see Fig. 7). This is because the normal component of the surface currents is not taken into account in the model of Kazantsev and Surdutovich [13]. Such approximation might be of common sense for infinitely thin and ideally conducting targets, but for real targets it is just a model. However, this model is not that bad, and real metal targets with a finite thickness and conductivity are satisfactory described with it [2], except for some special situations, e.g., when the radiation propagates under the angle about $90^{\circ}$ relative to the plane of a target. Good agreement between the theory based on the results of [13] and experimental data for $\gamma=2500$ was demonstrated in paper [41]. Discussion of validity for the model of infinitely thin and ideally conducting targets without normal component of surface currents is contained in the paper of Karlovets and Potylitsyn [42]. This imperfection is absent in the theories taking into account

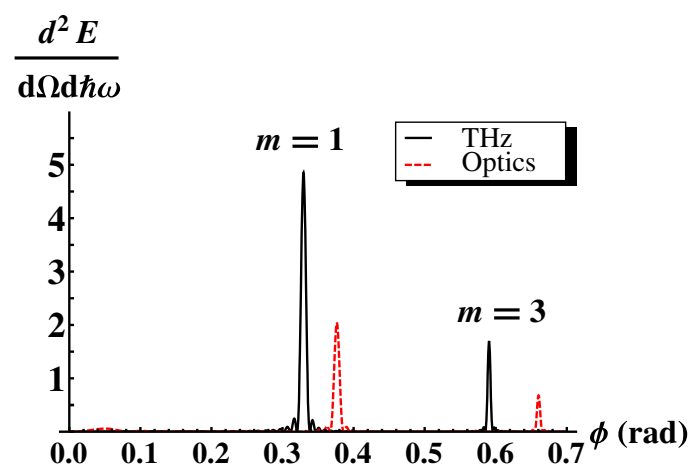

FIG. 7. The distribution of SPR over the conical surface, i.e., at $\theta=\arccos \left(\beta^{-1} \sin \alpha\right)$. Red dashed curve is the distribution for the optical frequency region. It is plotted using Eq. (35) for $\gamma=1710$ (electron energy of Mainz Microtron MAMI: $E=855 \mathrm{MeV}$ ), $d=9 \mu \mathrm{m}, \lambda=546 \mathrm{~nm}, h=127 \mu \mathrm{m}$. The black curve is the distribution for the $\mathrm{THz}$ frequencies, plotted using Eq. (35) for $\gamma=13, d=10 \mathrm{~mm}, \lambda=0.5 \mathrm{~mm}, h=0.5 \mathrm{~mm}$. For both curves $N=20, \alpha=\pi / 6, a=d / 2$.

the finite dielectric permittivity of the target more consistently-see our consideration above for $\mathrm{x}$-ray SPR, or papers $[14,19,20,32]$.

The distributions for the THz, optical and x-ray SmithPurcell radiation are plotted in Figs. 8(a) and 8(b) starting from the fifth order peaks, where angles satisfy Eq. (23). For parameters of Fig. 8(b) this region starts with value of the angle $\phi \approx 0.38 \mathrm{rad}$ and here there are the fifth and more spectral diffraction orders of $\mathrm{x}$-ray SPR. In the x-ray region [Fig. 8(b)] the directions corresponding these spectral orders are closer to the grating plane $x y$ than in optical and $\mathrm{THz}$ regions [Fig. 8(a)], which is in agreement with Eq. (38).

The multiplier $4 \sin ^{2}(a \varphi / 2)$ for a given diffraction order goes to $4 \sin ^{2}(\pi m a / d)$. If $a / d=l / m, l=1,2, \ldots$ this multiplier equals zero. To plot Figs. $6-8$ it is taken $a / d=1 / 2$, that is why one can see only odd peaks of SPR.

Now let us consider how the distribution of the radiation for separate SPR peaks (i.e., for separate spectral diffraction orders) depends on the angle $\alpha$. For example, Figs. 9(a) and 9(b) demonstrate this dependence for $\mathrm{THz}$, optical and $\mathrm{x}$-ray frequency regions for fifth and seventh SP peaks. Here we limit angles $\alpha$ to

$\alpha_{\max }=\min \left\{\arccos \left[\frac{d \beta}{2 m \lambda}\left(\frac{1}{\beta^{2} \gamma^{2}}+\frac{\lambda^{2} m^{2}}{d^{2}}\right)\right], \arcsin \beta\right\}$.

This inequality follows from Eq. (43) due to the simple fact that cosine is a limited function.

The sharp peaks in Fig. 9(b) are defined by the maximum of Eq. (48); the remainder of the graph after these peaks is caused by contribution of the evanescent waves discussed above. 

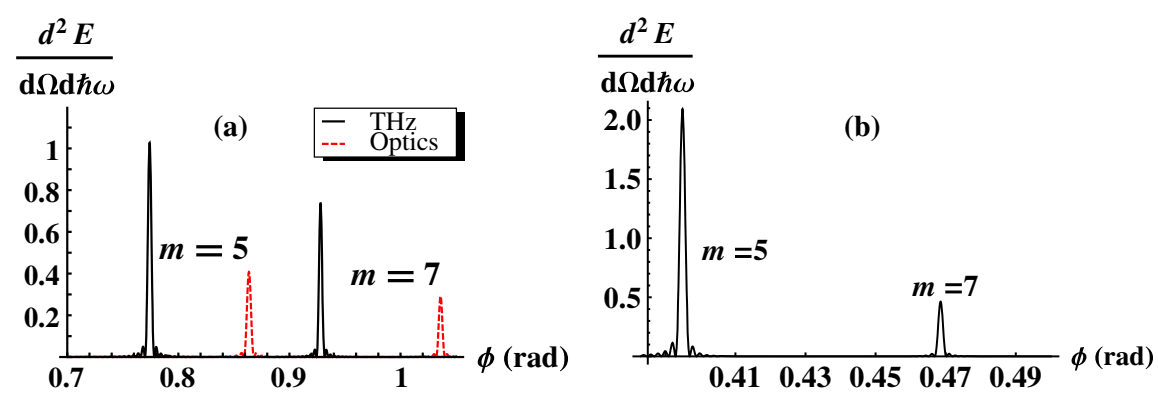

FIG. 8. The distribution of SPR over the conical surface, i.e., at $\theta=\arccos \left(\beta^{-1} \sin \alpha\right)$ [see Eqs. (28) and (35)]. (a) THz and optic frequency regions. Parameters are the same as in Fig. 7. (b) X-ray frequency region. Here $\gamma=4 \times 10^{4}$ (electron energy of FACET, SLAC: $E=28 \mathrm{GeV}$ ), $\hbar \omega_{p}=26.1 \mathrm{eV} \quad$ (beryllium), $\quad d=0.9 \mu \mathrm{m}, \quad a=0.45 \mu \mathrm{m}, \quad b=0.1 \mu \mathrm{m}, \quad \lambda=12 \mathrm{~nm}, \quad h=60 \mu \mathrm{m}$, $\varepsilon(\omega)=1-\omega_{p}^{2} / \omega^{2}, \alpha=\pi / 6, N=20$. The minimal value of the horizontal axis is the angle satisfying Eq. (23) or Eq. (47): $\phi \approx 0.38 \mathrm{rad}$.
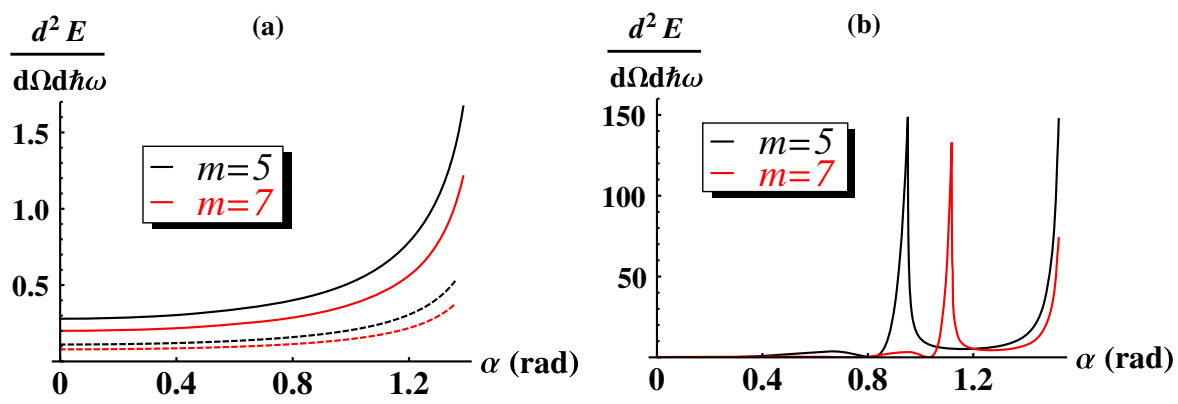

FIG. 9. The distribution of the radiation for fifth and seventh SPR peaks (diffraction orders) depending on $\alpha$ for (a) THz and optical and (b) x-ray frequency regions. The parameters are the same as in Fig. 7. It is taken into account that for radiation on the conical surface Eq. (43) is satisfied.

\section{Number and width of the Smith-Purcell peaks}

Now we consider some characteristics of the radiation following from Eq. (43).

First, the number of the diffraction orders is limited and values of minimal/maximal diffraction orders can be found from inequality:

$$
\begin{aligned}
& \frac{d}{\lambda}\left(\frac{\cos \alpha}{\beta}-\sqrt{1-\left(\frac{\sin \alpha}{\beta}\right)^{2}}\right) \\
& \leq m \leq \frac{d}{\lambda}\left(\frac{\cos \alpha}{\beta}+\sqrt{1-\left(\frac{\sin \alpha}{\beta}\right)^{2}}\right) .
\end{aligned}
$$

This inequality is analogous to the inequality known in optics [27]. In our case the number of diffraction orders $m$ depends on a direction of the particle velocity, see Fig. 10. Note that the range of angles $\alpha$ exists, in which there are no any diffraction orders-have a look at the area between $\alpha_{1}$ and $\alpha_{\max }$ in Fig. 10. Here $\alpha_{1}$ is the maximal angle for which only the first diffraction order exists; $\alpha_{\max }$ is the maximal admissible angle defined by Eq. (50).

The value of $\alpha_{\max }$ depends on the particle energy. For parameters of Fig. 10: $\alpha_{\max }=81.8^{\circ}$. By the way, the authors of paper [26], considering reflection of light from a grating under grazing incidence, also noticed that some extreme angle exists, and estimated them as $80^{\circ}$ and more, which is in agreement with our results.

Second, as we said above, besides the exponent and the ratio of squared sines in Eqs. (28) and (35) there is the multiplier depending on the angles of radiation. It leads to the shift of peaks on the conical surface. The

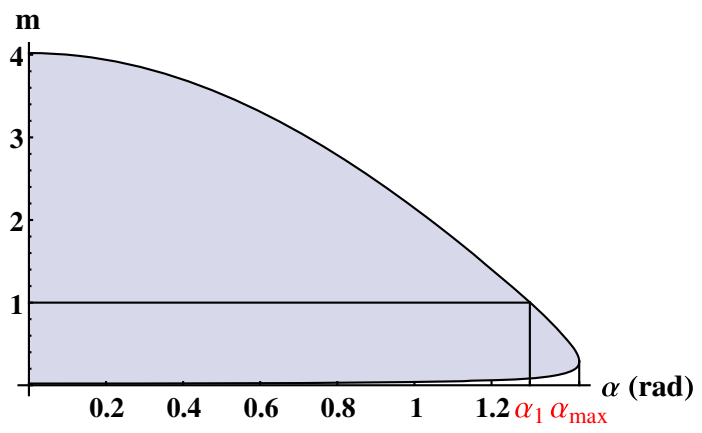

FIG. 10. A number of diffraction orders. Here $\alpha_{1}$ is the minimal particle velocity for which only the first diffraction order exists; $\alpha_{\max }$ is the maximal admissible angle [Eq. (50)]. It is plotted using Eq. (51) for $\gamma=7, d / \lambda=2$. 
maximal possible shift is equal to a half-width of a peak, which for

$$
\lambda /(d N) \ll \sqrt{1-\left(\beta^{-1} \sin \alpha\right)^{2}}
$$

proves to be

$$
\delta \phi=\frac{\lambda}{d N}\left[1-\frac{\sin ^{2} \alpha}{\beta^{2}}-\left(\frac{\cos \alpha}{\beta}-\frac{m \lambda}{d}\right)^{2}\right]^{-1 / 2} .
$$

In the general case, when Eq. (52) is not satisfied, the half-width is

$$
\begin{aligned}
\delta \phi= & \arcsin \left(\frac{1}{\beta} \frac{\cos \alpha-m \lambda \beta / d}{\sqrt{1-\left(\beta^{-1} \sin \alpha\right)^{2}}}\right) \\
& -\arcsin \left(\frac{1}{\beta} \frac{\cos \alpha-m \lambda \beta / d}{\sqrt{1-\left(\beta^{-1} \sin \alpha\right)^{2}}}-\frac{\lambda}{d N} \frac{1}{\sqrt{1-\left(\beta^{-1} \sin \alpha\right)^{2}}}\right) .
\end{aligned}
$$

For the optical region and parameters of the black curve in Fig. 7 (THz) for $m=1$ we have $\delta \phi=8.8 \mathrm{mrad}$ or for $m=7$ we have $\delta \phi=3.6 \mathrm{mrad}$; the red curve in Fig. 7 (optics): $\delta \phi=9.4 \mathrm{mrad}$ for $m=1$, or $\delta \phi=4.1 \mathrm{mrad}$ for $m=7$; in the x-ray region and parameters of Fig. 8(b) we have $\delta \phi=4 \mathrm{mrad}$ for $m=1$ and $\delta \phi=1.7 \mathrm{mrad}$ for $m=7$, correspondingly. In the x-ray range the peaks are considerably narrower.

Third, as we discussed above, the directions of the spectral orders of SPR are distributed over the conical surface defined by Eq. (40). Let us estimate possible deflection of the direction of radiation from the conical surface. For this one should deal with exponent

$$
\exp \left(-\frac{2 h \omega}{c \beta \gamma} \sqrt{1+\gamma^{2} \beta_{x}^{-2}\left(n_{y} \beta^{2}-\beta_{y}\right)^{2}}\right)
$$

and demand that

$$
n_{y} \leq \frac{\gamma^{-1} \beta_{x}+\beta_{y}}{\beta^{2}}
$$

The angle $\theta_{m}$ defining the opening angle of this cone is given by $\cos \theta_{m}=\beta_{y} / \beta^{2}$. Representing the angle of radiation as $\theta=\theta_{m}-\Delta \theta$ we have

$$
n_{y}=\frac{\gamma^{-1} \beta_{x}+\beta_{y}}{\beta^{2}}=\frac{\gamma^{-1} \beta_{x}}{\beta^{2}}+\cos \theta_{m} .
$$

Supposing $\Delta \theta \ll \theta_{m}$ we have

$$
n_{y}=\cos \left(\theta_{m}-\Delta \theta\right)=\cos \theta_{m}+\Delta \theta \sin \theta_{m} .
$$

It is easy to see from Eqs. (57) and (58) that

$$
\Delta \theta=\frac{\gamma^{-1}}{\sqrt{1-\gamma^{-2} / \cos ^{2} \alpha}} .
$$

Note that in case $\alpha=0$ Eq. (59) gives the usual width of angular distribution of SPR and DR: $\Delta \theta=(\beta \gamma)^{-1}$.

\section{Angular distribution of Smith-Purcell radiation}

Figure 10 demonstrates decrease in the number of peaks with growth of the incidence angle $\alpha$. Notice that the distributions of the radiated energy demonstrate rather sharp behavior as a function of the diffraction order $m$. On this account we shall consider the distributions for given $m$ separately.

To consider the changing of intensity of the peaks (spectral orders $m$ ) with growth of $\alpha$, it is convenient to deal with angular distribution. Integrating Eqs. (28) and (35) over the ranges of frequencies in which they are valid, we obtain two different angular distributions in general form:

$\frac{d E(\mathbf{n})}{d \Omega}=\frac{e^{2}}{c} \sin ^{2}\left(\frac{a}{d} \pi m\right) \frac{2 \pi N c \beta_{x}^{3} m^{2}}{d^{3}\left(1-n_{x} \beta_{x}-n_{y} \beta_{y}\right)^{5}} e^{-2 \rho_{m} h} P_{m}$,

where for $\omega_{m}>\omega_{p}$ in EUV/x-ray range

$$
\begin{aligned}
P_{m}= & F_{b m}\left(\varepsilon_{m}-1\right)^{2}\left|\left[\mathbf{n}^{\prime} \times \mathbf{n}^{\prime} \times\left(\frac{\mathbf{A}_{m}}{\rho_{m}}-i \mathbf{e}_{z}\right)\right]\right|^{2} \\
& \times\left|\rho_{m}-i \frac{\omega_{m}}{c} \sqrt{\varepsilon_{m}-1+n_{z}^{2}}\right|^{-2}
\end{aligned}
$$

and $\mathbf{n}^{\prime}$ is taken from the right part of Eq. (24); for the longwavelength region $\omega_{m}<\omega_{\text {optical }}\left(\omega_{\text {optical }}\right.$ is the upper bound of the visible range)

$$
\begin{aligned}
P_{m}= & \frac{1}{\beta_{x} \rho_{m}^{2}} \frac{\left(1-\beta_{y} n_{y}\right)}{\sqrt{1-n_{y}^{2}}}\left[\left(1-2 \beta_{y} n_{y}-\beta_{x}^{2}+\beta^{2} n_{y}^{2}\right)\right. \\
& \times\left(1+\frac{\beta_{x} \sqrt{1-n_{y}^{2}}}{1-\beta_{y} n_{y}}\right)\left(1-\frac{n_{x}}{\sqrt{1-n_{y}^{2}}}\right) \\
& \left.+\left(\beta_{y}-n_{y}\right)^{2}\left(1-\frac{\beta_{x} \sqrt{1-n_{y}^{2}}}{1-\beta_{y} n_{y}}\right)\left(1+\frac{n_{x}}{\sqrt{1-n_{y}^{2}}}\right)\right] .
\end{aligned}
$$

Here it is denoted 


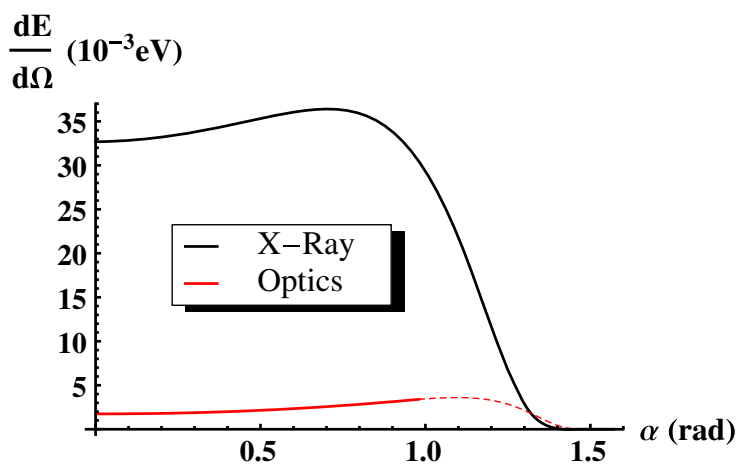

FIG. 11. Angular distribution of the fifth peak (fifth order) of SPR for optical region (red dashed curve) and for $\mathrm{x}$-ray region (black curve). It is plotted using Eqs. (60)-(63) for optical region $\gamma=1710$ (energy of Mainz Microtron MAMI), $d=9 \mu \mathrm{m}$, $h=127 \mu \mathrm{m}, \phi=0.9 \mathrm{rad}$; and for x-ray region $\gamma=4 \times 10^{4}$ (energy of SLAC), $\hbar \omega_{p}=26.1 \mathrm{eV}$ (beryllium), $d=0.9 \mu \mathrm{m}$, $h=60 \mu \mathrm{m}, \phi=0.3 \mathrm{rad}, \varepsilon(\omega)=1-\omega_{p}^{2} / \omega_{m}^{2}$. For both of the curves $N=20, m=5, a=d / 2, \theta=\arccos \left(\beta^{-1} \sin \alpha\right)$. The dashed line corresponds to the angles breaking the inequality $\omega_{m}<\omega_{\text {optical }}$ for $\lambda_{\text {optical }}=2 \pi c / \omega_{\text {optical }}=380 \mathrm{~nm}$.

$$
\begin{aligned}
\varepsilon_{m} & =1-\frac{\omega_{p}^{2}}{\omega_{m}^{2}}, \quad \omega_{m}=\frac{2 \pi m c \beta_{x}}{d\left(1-n_{x} \beta_{x}-n_{y} \beta_{y}\right)}, \\
\rho_{m} & =\frac{\omega_{m}}{c \beta \gamma} \sqrt{1+\gamma^{2} \beta_{x}^{-2}\left(n_{y} \beta^{2}-\beta_{y}\right)^{2}}, \\
\mathbf{A}_{m} & =\frac{\omega_{m}}{c \beta_{x}}\left(1-n_{y} \beta_{y}-\beta_{x}^{2}, \beta_{x}\left(n_{y}-\beta_{y}\right), 0\right), \\
F_{b m} & =\left|1-\exp \left(-b \rho_{m}+i b \frac{\omega_{m}}{c} \sqrt{\varepsilon_{m}-1+n_{z}^{2}}\right)\right|^{2} .
\end{aligned}
$$

The angular distribution for the fifth order of SPR is shown in Fig. 11. One can see that up to some definite value of angle the intensity increases, after this decreases, and this behavior is the same for two different physical models valid for two different ranges of frequencies. However, the theory may lose its applicability at the angles less than that corresponding to maximum of the radiation, and in this case the function can increase over all the range of permit angles - see the red curve in Fig. 11. The distributions in Fig. 11 are plotted for two different gratings and parameters of beam for optical and lower frequencies and for UV and $\mathrm{x}$-ray frequencies, respectively.

\section{SUMMARY AND CONCLUDING REMARKS}

The theory of Smith-Purcell radiation has been constructed for x-ray, EUV, optical, and THz ranges. For highfrequency range (EUV/x-rays) the analytical expressions have been obtained directly from Maxwell's equations and are presented here for the first time. For the lower frequencies of optical and $\mathrm{THz}$ ranges we constructed the theory of SPR proceeding from the known generalization [22] of one of the most powerful approaches in the theory of diffraction radiation [13]. Our results are valid for arbitrary energies of the charged particles, from nonrelativistic to ultrarelativistic. We stress the conical effect in SPR - the strong spatial redistribution of the SPR peaks (spectral orders) over a conical surface. This effect was first investigated by Haeberle et al. [15] proceeding from the well-known van den Berg approach [10], which, however, does not always work well for ultrarelativistic energies [16], and demands additional difficult numerical calculations into the bargain. In contrast with that we suggest a fully analytic description displaying important new features of radiation.

The spectral and angular distributions of SPR show strong dependence on the angle $\alpha$ between a particle velocity and the normal to the rulings direction. Spatial distributions of the SPR peaks for different spectral ranges are proved to have a lot in common; on the other hand, there are also some differences, which were analyzed in detail in Sec. V. The angular distribution of SPR as a function of $\alpha$ has a maximum (Fig. 11); the spectral and angular distribution in the EUV/x-ray region has a sharp maximum [Fig. 9(b)] owing to the contribution of evanescent waves: see the discussion after Eqs. (25) and (48). We cannot assert that the same behavior-sharp peaks due to evanescent waves-will occur in other spectral ranges, but for nonmetal targets we believe it to be so.

Redistribution of radiation from the plane to the cone occurs when $\alpha=0$ becomes $\alpha \neq 0$ and therefore will play a vital part for divergent beams. Modern and prospective highbrightness electron beams having small transverse dimensions, the beams from laser-plasma sources, and nonrelativistic beams-all these need allowance to be made for divergence in order to construct a correct theory. Also, the distribution of radiation caused by the interaction of charged particles with photonic crystal (including targets made of metamaterial, which often is a crystal) should be strongly influenced by the conical effect considered here in detail.

EUV and X-ray SPR have not been observed experimentally so far, but we are sure that shortwave DR and SPR have great potential for submicron noninvasive beam diagnostics of high-brightness ultrarelativistic beams. Also, our theory of $\mathrm{x}$-ray SPR is valid in the water-window range $(\lambda=4.47-2.36 \mathrm{~nm})$ where $\mathrm{x}$-ray radiation is not absorbed by water vapor; development of radiation sources with tunable radiation spectrum in this frequency range is important in practical applications in medicine and biology. Also, noninvasive beam diagnostics in this frequency range is very attractive for ultrarelativistic super high-brightness beams. For example, the size of the focal spot in ILC is expected to be about $8 \mathrm{~nm}$, needing detection with an accuracy of some nanometers, which is not provided by existing techniques and demands developing short-wave schemes of diagnostics.

Thus, our results may be of importance for beam diagnostic systems and for designing of new sources of electromagnetic radiation in different frequency ranges, including free-electron lasers. 


\section{ACKNOWLEDGMENTS}

We are grateful to Professor A. P. Potylitsyn for stimulating discussions. The work was supported by the Ministry of Education and Science of the Russian Federation, Grant No. 3.1110.2014/K.

[1] S. J. Smith and E. M. Purcell, Phys. Rev. 92, 1069 (1953).

[2] A. P. Potylitsyn, M. I. Ryazanov, M. N. Strikhanov, and A. A. Tishchenko, Diffraction Radiation from Relativistic Particles (Springer, Berlin, 2011).

[3] P. Rullhusen, X. Artru, and P. Dhez, Novel Radiation Sources Using Relativistic Electrons (World Scientific, Singapore, 1998).

[4] A. A. Ponomarenko, M. I. Ryazanov, M. N. Strikhanov, and A. A. Tishchenko, Nucl. Instrum. Methods Phys. Res., Sect. B 309, 223 (2013); W. Knulst, M. J. van der Wiel, O. J. Luiten, and J. Verhoeven, Appl. Phys. Lett. 83, 4050 (2003); A. Smirnov, A high performance, FIR radiator based on laser driven e-gun, in Photonics Research Developments, edited by V.P. Nilsson (Nova Science Publishers, Inc., Hauppauge, New York, 2008).

[5] I. V. Konoplev, A. J. MacLachlan, C. W. Robertson, A. W. Cross, and A. D. R. Phelps, Phys. Rev. A 84, 013826 (2011).

[6] L. Schachter and A. Ron, Phys. Rev. A 40, 876 (1989); D. Li, M. Hangyo, Y. Tsunawaki, Z. Yang, Y. Wei, S. Miyamoto, M. R. Asakawa, and K. Imasaki, Appl. Phys. Lett. 100, 191101 (2012); J. Gardelle, P. Modin, and J. T. Donohue, Phys. Rev. Lett. 105, 224801 (2010); J. D. Jarvis, H. L. Andrews, and C. A. Brau, Phys. Rev. ST Accel. Beams 13, 020701 (2010).

[7] F. J. Garcia de Abajo, Rev. Mod. Phys. 82, 209 (2010); H. L. Andrews et al., Phys. Rev. ST Accel. Beams 17, 052802 (2014).

[8] P. Karataev, S. Araki, R. Hamatsu, H. Hayano, T. Muto, G. Naumenko, A. Potylitsyn, N. Terunuma, and J. Urakawa, Phys. Rev. Lett. 93, 244802 (2004); A. Cianchi, V. Balandin, M. Castellano, E. Chiadroni, L. Catani, N. Golubeva, K. Honkavaara, G. Kube, and M. Migliorati, New J. Phys. 16, 113029 (2014).

[9] V. P. Shestopalov, The Smith-Purcell Effect (Nova Science Publishers, Commack, NY, 1993).

[10] P. M. van den Berg, J. Opt. Soc. Am. 63, 689 (1973) (in Russian).

[11] J. H. Brownell, J. Walsh, and G. Doucas, Phys. Rev. E 57, 1 (1998); J. H. Brownell and G. Doucas, Phys. Rev. ST Accel. Beams 8, 091301 (2005).

[12] A. P. Potylitsyn, Nucl. Instrum. Methods Phys. Res., Sect. B 145, 60 (1998).

[13] A. P. Kazantsev and G. I. Surdutovich, Sov. Phys. Dokl. 7, 990 (1963).

[14] D. V. Karlovets, J. Exp. Theor. Phys. 113, 27 (2011).

[15] O. Haeberle, P. Rullhusen, J.-M. Salome, and N. Maene, Phys. Rev. E 55, 4675 (1997).

[16] G. Kube et al., Phys. Rev. E 65, 056501 (2002).

[17] A. A. Tishchenko, A. P. Potylitsyn, and M. N. Strikhanov, Phys. Rev. E 70, 066501 (2004).
[18] A. A. Tishchenko, M. N. Strikhanov, and A. P. Potylitsyn, Nucl. Instrum. Methods Phys. Res., Sect. B 227, 63 (2005).

[19] A. A. Tishchenko, A. P. Potylitsyn, and M. N. Strikhanov, Phys. Lett. A 359, 509 (2006).

[20] D. Yu. Sergeeva, A. A. Tishchenko, and M. N. Strikhanov, Nucl. Instrum. Methods Phys. Res., Sect. B 309, 189 (2013).

[21] D. Yu. Sergeeva, M. N. Strikhanov, and A. A. Tishchenko, in Proceedings of the 4th International Particle Accelerator Conference, IPAC-2013, Shanghai, China, 2013 (JACoW, Shanghai, China, 2013), p. 616.

[22] N. Potylitsina-Kube and X. Artru, Nucl. Instrum. Methods Phys. Res., Sect. B 201, 172 (2003).

[23] S. Yamaguti, J. Inoue, O. Haeberle, and K. Ohtaka, Phys. Rev. B 66, 195202 (2002); N. Horiuchi et al., Phys. Rev. E 74, 056601 (2006); A. Yanai and U. Levy, Opt. Express 20, 18515 (2012).

[24] M.-E. Couprie et al., in Proceedings of 36th International Free Electron Laser Conference FEL 2014, Basel, Switzerland, 2014 (JACoW, Basel, 2014), p. 569.

[25] G. H. Spencer and M. V. R. K. Murty, J. Opt. Soc. Am. 52, 672 (1962).

[26] M. Neviere, D. Maystre, and W. R. Hunter, J. Opt. Soc. Am. 68, 1106 (1978).

[27] E. N. Hogert, M. A. Rebollo, and N. G. Gaggioli, Optics Laser Technol. 23, 341 (1991).

[28] B. Dolgoshein, Nucl. Instrum. Methods Phys. Res., Sect. A 326, 434 (1993).

[29] L. Durand, Phys. Rev. D 11, 89 (1975).

[30] A. A. Tishchenko and D. Yu. Sergeeva, in Proceedings of 36th International Free Electron Laser Conference FEL 2014, Basel, Switzerland, 2014 (JACoW, Basel, 2014), p. 384.

[31] D. Yu. Sergeeva and A. A. Tishchenko, in Proceedings of 36th International Free Electron Laser Conference FEL 2014, Basel, Switzerland, 2014 (JACoW, Basel, 2014), p. 378.

[32] M. V. Shevelev and A. S. Kon'kov, J. Exp. Theor. Phys. 118, 501 (2014).

[33] M. I. Ryazanov, M. N. Strikhanov, and A. A. Tishchenko, J. Exp. Theor. Phys. 99, 311 (2004); I. P. Ivanov and D. V. Karlovets, Phys. Rev. A 88, 043840 (2013).

[34] M. J. Moran, Phys. Rev. Lett. 69, 2523 (1992).

[35] L. M. Brekhovskikh, Usp. Fiz. Nauk 38, 1 (1949) (in Russian).

[36] A. A. Tishchenko, M. N. Strikhanov, and A. P. Potylitsyn, $U V$ and $x$-ray Smith-Purcell radiation, in Book of Abstracts of VIII International Symposium "Radiation from Relativistic Electrons in Periodic Structures," RREPS-2009, Zvenigorod, Russia, 2009, p. 50.

[37] V. V. Syshchenko and N. F. Shulga, J. Surf. Invest.: X-Ray, Synchrotron Neutron Tech. 5, 392 (2011).

[38] S. J. Glass and H. Mendlowitz, Phys. Rev. 174, 57 (1968).

[39] O. Haeberle, P. Rullhusen, J.-M. Salome, and N. Maene, Phys. Rev. E 49, 3340 (1994).

[40] D. V. Karlovets and A. P. Potylitsyn, Phys. Rev. ST Accel. Beams 9, 080701 (2006).

[41] T. Muto, S. Araki, R. Hamatsu, H. Hayano, T. Hirose, P. Karataev, G. Naumenko, A. Potylitsyn, and J. Urakawa, Phys. Rev. Lett. 90, 104801 (2003).

[42] D. V. Karlovets and A. P. Potylitsyn, Phys. Lett. A 373, 1988 (2009). 